\title{
Enhancing oil production in Arabidopsis through expression of a ketoacyl-ACP synthase domain of the PUFA synthase from Thraustochytrium
}

\author{
Xi Xie, Dauenpen Meesapyodsuk and Xiao Qiu*
}

\begin{abstract}
Background: Plant seed oil is an important bioresource for human food and animal feed, as well as industrial bioproducts. Therefore, increasing oil content in seeds has been one of the primary targets in the breeding programs of oilseed crops. Thraustochytrium is a marine protist that can produce a high level of very long-chain polyunsaturated fatty acids (VLCPUFAs) using a PUFA synthase, a polyketide synthase-like fatty acid synthase with multiple catalytic domains. Our previous study showed that a KS domain from the synthase could complement an Escherichia coli mutant defective in $\beta$-ketoacyl-ACP synthase I (FabB) and increase the total fatty acid production. In this study, this KS domain from the PUFA synthase was further functionally analyzed in Arabidopsis thaliana for the capacity of oil production.
\end{abstract}

Results: The plastidial expression of the KS domain could complement the defective phenotypes of a KASI knockout mutant generated by CRISPR/Cas9. Seed-specific expression of the domain in wild-type Arabidopsis significantly increased seed weight and seed oil, and altered the unsaturation level of fatty acids in seeds, as well as promoted seed germination and early seedling growth.

Conclusions: The condensation process of fatty acid biosynthesis in plants is a limiting step, and overexpression of the KS domain from a PUFA synthase of microbial origin offers a new strategy to increase oil production in oilseed plants.

Keywords: PUFA synthase, KS domain, Oil content, Seed weight, Thraustochytrium

\section{Background}

Plant seed oil is an important bioresource not only for food and feed, but also for industrial bioproducts such as biofuel and biopolymers potential for replacing nonrenewable petro-polymers and petro-fuels [1-3]. Therefore, increasing oil content in seeds has long been one of the primary targets in the breeding programs of oilseed crops. In the past few years, the success has been made through various molecular breeding approaches, such as manipulation of transcriptional factors in carbon

*Correspondence: xiao.qiu@usask.ca

Department of Food and Bioproduct Sciences, University

of Saskatchewan, Saskatoon, SK S7N 5A8, Canada partitioning, regulation of the biosynthetic process of lipids, and overexpression of key enzymes in fatty acid biosynthesis and assembly. For instance, overproduction of the regulator WRINKLED1 in Arabidopsis thaliana resulted in enlarged seeds and $10-40 \%$ oil increase [4]. Overexpression of a yeast glycerol-3-phosphate dehydrogenase for the formation of glycerol-3-phosphate at the starting point of the Kennedy pathway boosted oil content by $40 \%$ in the seeds of oilseed rape [5]. Seedspecific expression of acyl-CoA:diacylglycerol transferase 1 (DGAT1) for the biosynthesis of triacylglycerol (TAG) enhanced oil content by about $20 \%$ in $A$. thaliana [6]. In addition, increased activity of plastidial enzymes such as biotin carboxyl carrier protein isoform 2 (BCCP2) in 
acetyl-coenzyme carboxylase (ACCase), pyruvate transporter (BASS2) and purple acid phosphatase 2 could also raise oil levels in oilseeds [7-9].

In plant, fatty acid biosynthesis occurs in plastids where acetyl-CoA carboxylase (ACCase) catalyzes the formation of malonyl-CoA from acetyl-CoA initially, and then, a type II fatty acid synthase (FAS) complex uses malonyl thioester as an extender for the synthesis of long-chain saturated fatty acids such as 16:0 and 18:0 through a repetitive cycle of four catalytic reactions: condensation, keto-reduction, dehydration and enoyl-reduction [10]. After synthesis, these two long-chain fatty acids are often desaturated by a soluble acyl-ACP $\Delta 9$-desaturase in the stroma of plastids giving 16:1-9 and 18:1-9. Subsequently, these saturated and monounsaturated longchain fatty acids are exported to cytosol where they can be further modified by desaturations to introduce more double bonds or elongations to extend the chain length. With few exceptions [11], higher plants do not possess capacity to synthesize very long-chain polyunsaturated fatty acids (VLCPUFAs) with the chain length of $20 \mathrm{C}$ or more and the double bonds of two or more.

De novo biosynthesis of VLCPUFAs occurs mainly in microorganisms where their biosynthesis goes through either an aerobic pathway catalyzed by desaturases and elongases or an anaerobic pathway catalyzed by a polyunsaturated fatty acid (PUFA) synthase. Thraustochytrium is a protist producing a high level of a nutritionally important VLCPUFA docosahexaenoic acid (DHA, 22:6-n3). Biosynthesis of this fatty acid is catalyzed by a PUFA synthase, a polyketide synthase-like mega-enzyme $[12,13]$. Structurally, the PUFA synthase shares similarity to both type I and type II fatty acid synthases in catalytic domains and motifs. Functionally, both PUFA synthase, and type I and II fatty acid synthases catalyze the synthesis of fatty acids through similar reiterative reactions such as condensation, keto-reduction, dehydration and enoyl-reduction using acyl-ACPs as substrates [14-16]. The PUFA synthase in Thraustochytrium comprises three large subunits, each with multiple catalytic domains. However, the exact functions of these domains remain poorly understood. Our previous study showed ketoacyl-ACP synthase (KS) domains from the PUFA synthase could functionally complement the defective phenotype of two E. coli mutants of $\beta$-ketoacyl-ACP synthases. Particularly, overexpression of one KS domain from subunit-B (KS-B) resulted in accumulation of a high level of total fatty acids as well as unsaturated fatty acids in E. coli [17]. The aim of this study was to extend our previous research on this domain to model oilseed plant Arabidopsis for further functional analysis. The results showed that the KS domain was functional in the plant and able to complement the defective phenotype of an
Arabidopsis KASI knockout mutant generated by clustered regularly interspaced short palindromic repeats/ CRISPR-associated protein-9 nuclease (CRISPR/Cas9), and the seed-specific expression of this domain in wildtype Arabidopsis significantly enhanced seed weight and seed oil and promoted seed germination and seedling growth. These results emphasize that the condensation process of the fatty acid biosynthesis in plants is a limiting step and the enhanced activity by overexpression of the KS domain from a PUFA synthase of microbial origin offers an attractive biotechnological approach to increase oil content in oilseed plants.

\section{Results}

Sequence comparison of the KS domain with $\beta$-ketoacyl-ACP synthase I from plants

The PUFA synthase from Thraustochytrium was comprised of three subunits encoded by three large open reading frames (ORFs), each with multiple catalytic domains predicted by the presence of characteristic active site sequence motifs or residues [13]. One KS domain (KS-B) from residue 1 to 469 was identified in subunit-B of the PUFA synthase. Comparison of this KS domain with known plant $\beta$-ketoacyl-ACP synthase I (KASI) (Fig. 1) showed that it shared only about $20 \%$ amino acid sequence identity to plant KAS I from $A$. thaliana, Jatropha curcas, Nicotiana tabacum, Oryza sativa throughout the entire sequence. However, key residues in the active site such as a cysteine $(\mathrm{C})$, two histidines $(\mathrm{H})$ and a lysine (K) essential for the decarboxylation during the condensation process were completely conserved among these sequences. In addition, a glycine $(G)$ residue presumably located at the accepting entrance of the substrate-binding tunnel and two threonine $(\mathrm{T})$ residues likely involved in hydrogen bonding with the ACP phosphopantetheine moiety were also highly conserved. Furthermore, a glycine-rich motif located in the C-terminus contributing to the formation of an oxyanion hole for decarboxylation reaction was also observed among these sequences [18-20].

\section{Generation of the constructs disrupting endogenous KASI and expressing the KS domain in Arabidopsis}

To functionally analyze the KS domain in Arabidopsis, two binary plasmids were constructed. The first one was used to disrupt endogenous KASI genes, and the other was used to express the KS domain as a standalone enzyme from the PUFA synthase. To generate a construct to disrupt the KASI gene in Arabidopsis, two sets of overlapping sgRNA primer sequences targeting the conserved coding region of the gene were synthesized. The adaptor formed from each set of primers with a sgRNA sequence was inserted behind an Arabidopsis U3 


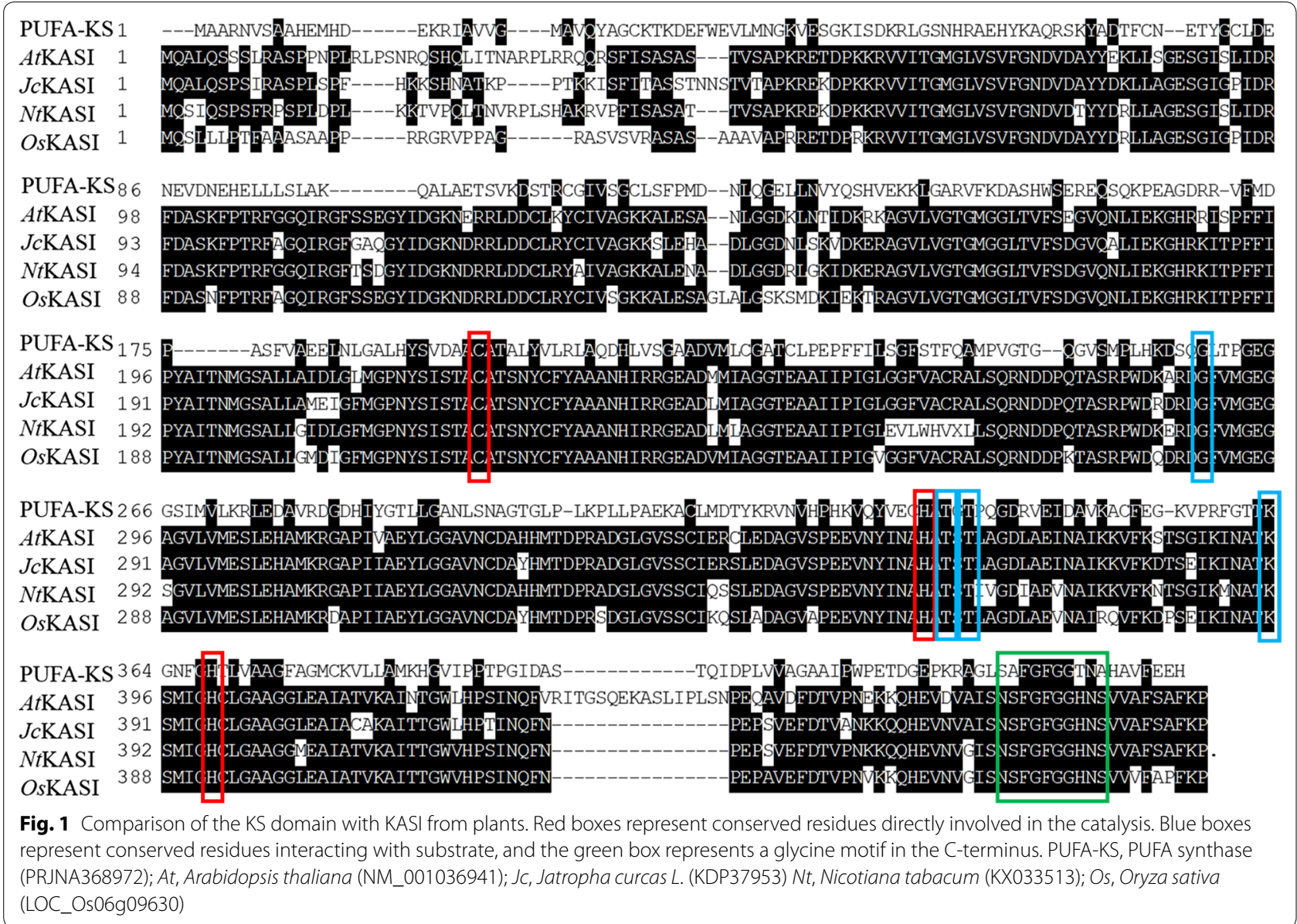

promoter in an intermediate plasmid pLYsgRNA-AtU3d, giving a sgRNA expression cassette. Two sgRNA expression cassettes were amplified with universal primer sets [21] and assembled into a CRISPR/Cas9 binary vector pYLCRISPR/Cas9- $\mathrm{P}_{\text {ubi }}-\mathrm{B}$ by a single golden gate ligation, giving the KASI-disrupting plasmid. Besides the two sgRNA expression cassettes for guiding the disruption of targeted genes, this construct was also comprised of a cassette expressing Cas 9 under a constitutive ubiquitin promoter for cutting the targeted sites, a cassette expressing a herbicide resistant Bar gene under a constitutive $35 \mathrm{~S}$ promoter for selecting transgenic plants, and a cassette expressing a modified green fluorescent protein (eGFP) from Aequorea victoria under a seed-specific napin promoter for screening transgenic seeds (Fig. 2a).

The second binary plasmid was constructed to express the KS domain in Arabidopsis. It was comprised of three expression cassettes. The first cassette was to express the KS domain as a standalone protein with a chloroplast transit peptide from Arabidopsis fused to the $\mathrm{N}$-terminus under the control of the seed-specific promoter. The second cassette was to express a kanamycin-resistance gene under the control of a constitutive promoter for selecting transgenic plants. The third cassette was to express a red fluorescent protein gene (DsRed2) from Discosoma under the seed-specific promoter for screening transgenic seeds (Fig. 2b).

After the confirmation of the structures of the two constructs by sequencing, they were introduced into Arabidopsis by floral dipping through an Agrobacterium tumefaciens-mediated transformation approach. Transgenic seeds with fluorescence were selected under a fluorescent microscope and grown to the next generation for further analysis.

\section{Complementation of Arabidopsis kasI mutant by the KS domain}

KASI is essential for the biosynthesis of fatty acids in plant, and thus, knockout mutants of the gene for this enzyme in Arabidopsis [22] might not be easily obtained for the functional complementation test. Therefore, for the complementation assays of the KS domain in Arabidopsis, two approaches were attempted. The first approach was to generate a kasI mutant Arabidopsis 

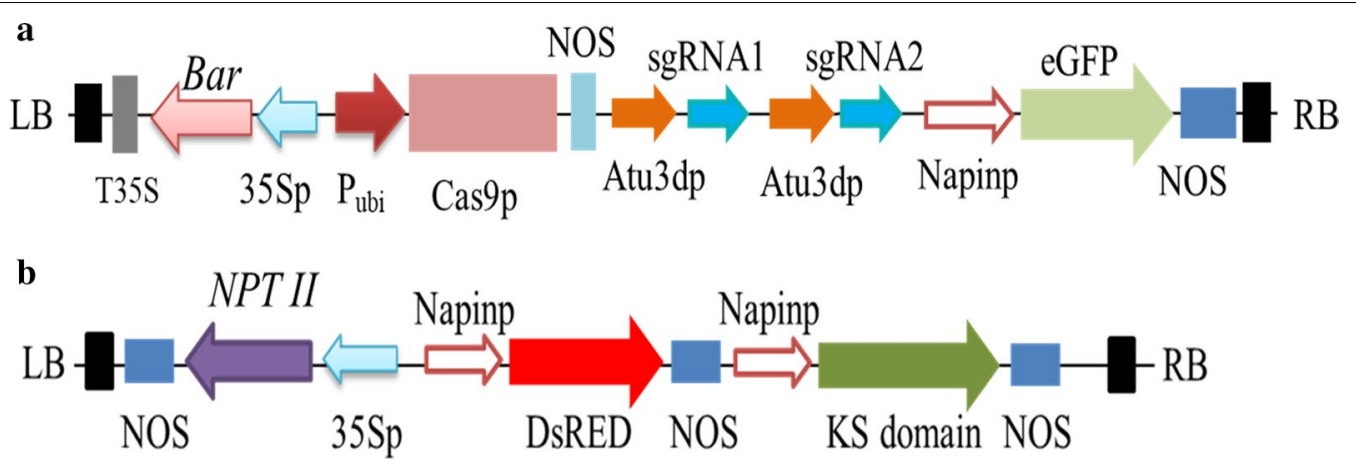

Fig. 2 Schematic representation of the T-DNA region of two binary vectors. a The T-DNA for of AtKASI-disrupting plasmid. b The T-DNA region of the KS domain expression plasmid

using a CRISPR/Cas9 technique, and then express the domain in the mutant, if it was amenable. The second approach was to express the domain first in wild-type Arabidopsis and then to disrupt the KASI by the CRISPR/ Cas9 technique.

To knock out KASI in Arabidopsis, the disrupting construct was first used to transform wild-type $A$. thaliana. The transgenic seeds were selected under a fluorescent microscope and grown to the next generation for genotyping and phenotyping. Genotyping transgenic T2 plants by sequencing the amplicons of targeted genes identified three homozygous lines with deletion mutation between two targeted sites of the KASI gene (kasI1 , kasI-4 and kasI-11). All these lines exhibited similar abnormal growth: smaller and shorter seedlings at the early growth stage and semi-dwarf at the late growth stage (Fig. 3), compared to the wild type. The similar phenotype was previously observed in a kasI mutant generated by a T-DNA insertion [22]. Due to the severe growth defect, re-transformation of the mutant with the KSexpression construct for the complementation test was not successful.

As the first approach for the complementation assay was unsuccessful, we then resorted the second approach by expressing the KS domain in Arabidopsis first and then disrupting KASI under the KS-expressed background. A transgenic plant expressing the KS domain obtained using the second construct for the first transformation was re-transformed with the disrupting construct of CRISPR/Cas9, and transgenic seeds were selected on both green (by eGFP) and red (by DsRed) fluorescence signals and grown to the next generation for genotyping and phenotyping. Genotyping analysis identified two mutation lines (KS/kasI-9, KS/kasI-15) with insertion mutation in the target gene. One had a single nucleotide insertion, while the other had a two-nucleotide insertion close to the targeted sites; both resulted in open reading frame-shifting mutations. Phenotypic observations showed that the two transgenic plants had comparable growth and development to the wild type, and no abnormal phenotypes were detected under the growth condition (Fig. 3).

To confirm the expression of the KS domain in a complementation line, reverse transcription PCR (RT-PCR) was performed using total RNAs isolated from 7th to 8th rosette leaves and developing siliques at 14 days after flowering [23]. A semi-quantitative PCR analysis showed that no expression of the KS domain was observed in the untransformed Arabidopsis control, but the expression of the KS domain could be obviously detected in both leaves and developing siliques of the complementation line (Fig. 4a). A quantitative PCR analysis showed that the transcription level of the KS domain in developing siliques was tenfold higher than that in leaves (Fig. 4b). This result indicated that the KS domain guided by a seed-specific napin promoter indeed possessed significantly higher expression in developing seeds than leaves, but it did express in leaves, providing the function for complementing the KASI defective phenotype in Arabidopsis.

\section{Seed-specific expression of the KS domain in wild-type Arabidopsis}

To examine the impact of the KS domain in fatty acid biosynthesis and oil accumulation in Arabidopsis, the second construct expressing the domain under the seed-specific promoter was introduced into wild-type Arabidopsis. Transgenic seeds were selected on a red fluorescence signal and propagated to the next generation. To confirm the expression of the KS domain, the total RNAs were isolated from the developing siliques of four transgenic lines (OE-6, OE-8, OE-13 and OE-16) and reverse-transcribed to cDNAs. A semi-quantitative PCR analysis of the cDNAs showed that the expression 
a

23DAG
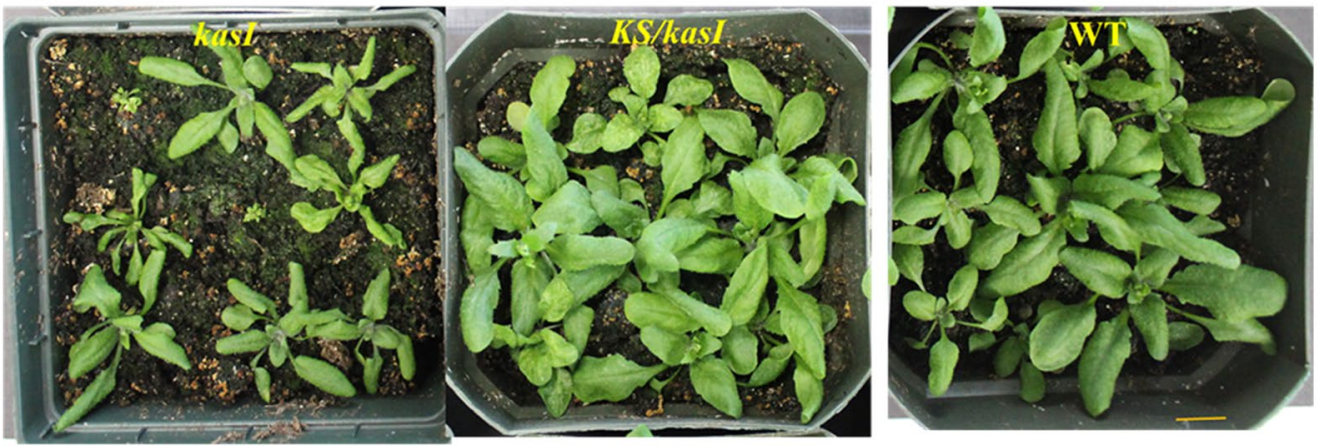

b

\section{DAG}

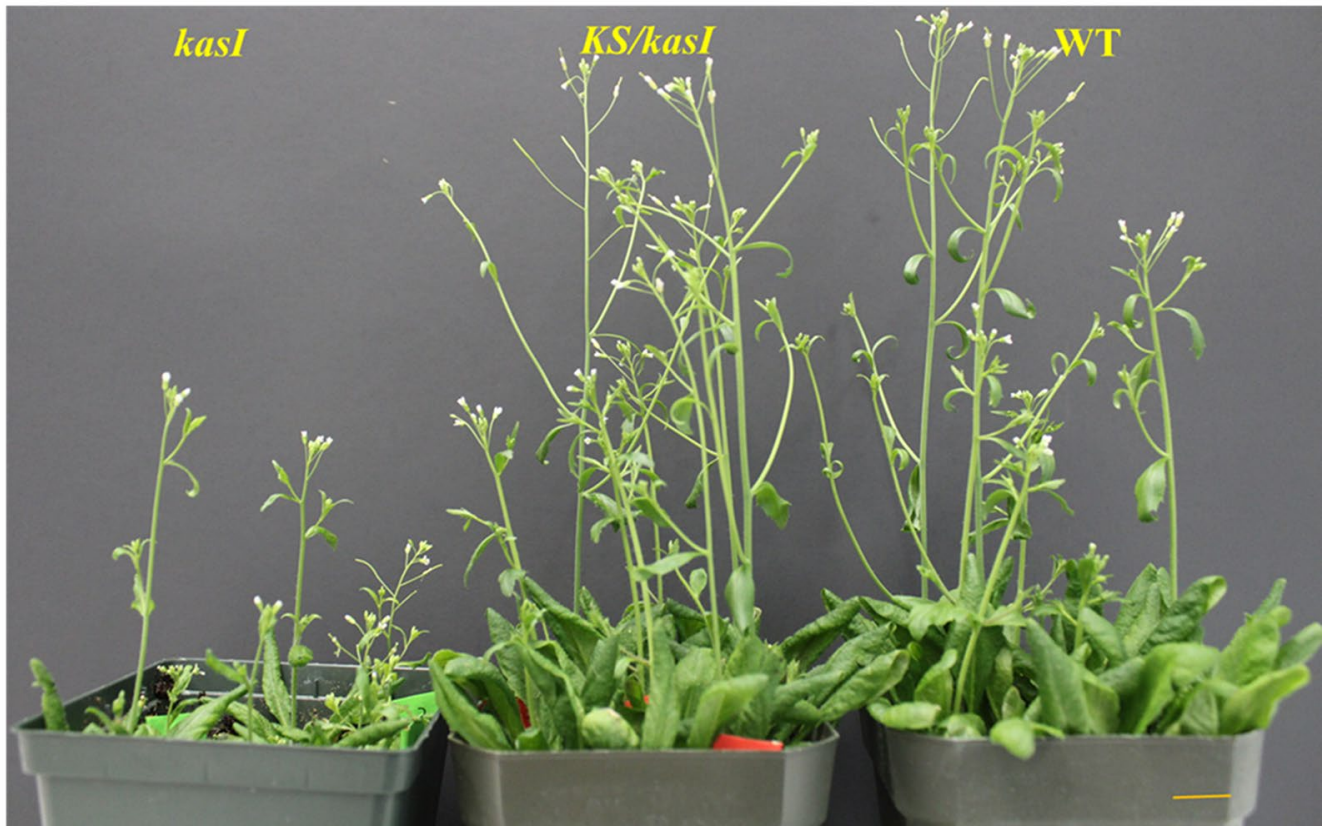

Fig. 3 Complementation of the kas/ mutant in Arabidopsis. a Growth phenotypes of kas/ mutant line, kas/ complementation line (KS/kas/) at 23 days after growing (DAG). Bars $=2 \mathrm{~cm}$. b Growth phenotypes of kas/ mutant line, kas/ complementation line (KS/kasl) at 35 DAG. Bars $=2 \mathrm{~cm}$

of the KS domain was much higher than that of a housekeeping gene Actin-2 (Fig. 5a). A quantitative PCR analysis showed that the transcription levels of the KS domain were highly varied among the four lines. The expression level of the KS domain in OE-6 was the highest, followed by $\mathrm{OE}-16$ and $\mathrm{OE}-13$, and $\mathrm{OE}-8$ had the least expression (Fig. 5b). Subsequently, the oil and mass of the seeds in these four transgenic lines were measured. As shown in Fig. 6, all the transgenic lines accumulated more oil relative to the wild-type control and the amount of oil in the mutant complementation line lied between those in the control and transgenic lines. The amount of oil per seed in OE-6, OE-16, OE-13 and OE-8 was increased by $80 \%$,
$77 \%, 69 \%$ and $48 \%$, respectively, over the control. In particular, the level of monounsaturated fatty acids (MUFAs) such as C18:1 and C20:1 was significantly higher in these transgenic lines over the wild type, the indicator of the seed triacylglycerol production seen previously [24]. In addition, the decrease in total saturated fatty acids and the increase in total unsaturated fatty acids were also observed in the transgenic lines (Table 1). Intriguingly, the transgenic seeds were also significantly heavier, representing 1.4, 1.2, 1.4 and 1.5-fold increase, respectively, relative to the wild type. Percentages of oil content in these transgenic lines exhibited increases by $14 \%$ to $28 \%$ over the control. Nevertheless, no significant difference 
a

\section{WT-L WT-S CE-L CE-S}

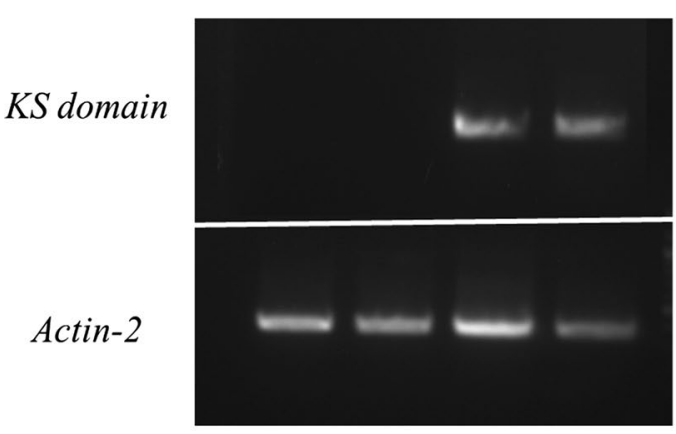

b

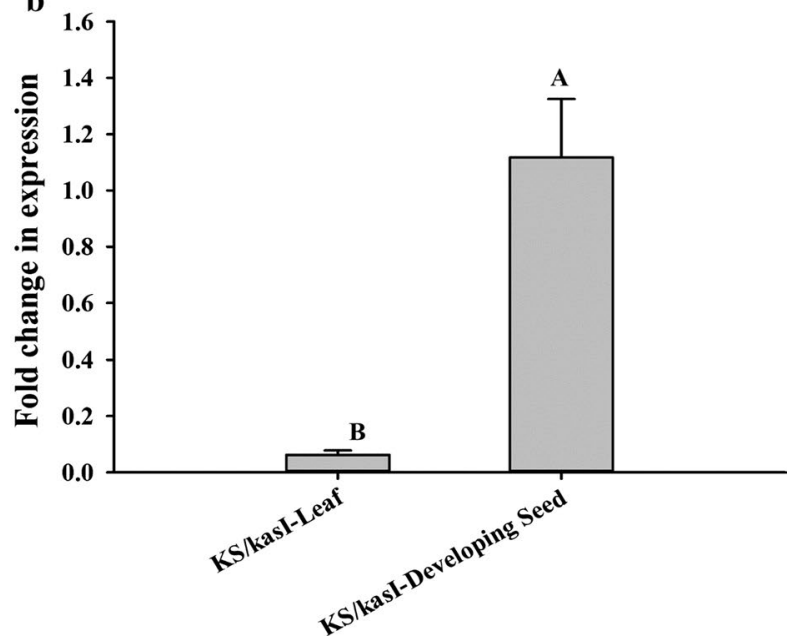

Fig. 4 Transcriptional analysis of the KS domain in leaves and developing siliques of a complementation line. a Expression of the KS domain and AtActin-2 in leaves and developing siliques of KS/kasl-9 analyzed by semi-quantitative RT-PCR. $\mathbf{b}$ Expression of the KS domain in leaves and developing siliques of KS/kasl-9 analyzed by quantitative RT-PCR. KS/kas/ kas/ mutant complementation line, WT-L leaves of wild type, WT-S siliques of wild type, CE-L leaves of complementation line, CE-S siliques of complementation line. Values are reported as the means of three biological replicates along with standard deviation. The means with the same letters are not statistically significantly different. Statistical analysis of the results was conducted using the one-way analysis of variance $(P<0.05)$

KS domain
Actin-2
Fig. $\mathbf{5}$ Transcriptional analysis of the $K S$ domain in developing siliques of overexpression lines. a Expression of the $K S$ domain and AtActin-2 in the
developing siliques of $K S / W T$ analyzed by semi-quantitative RT-PCR. $\mathbf{b}$ Expression of the $K S$ domain in leaves and developing siliques of $K S / W T$
analyzed by quantitative RT-PCR. $W T$ wild type, OE overexpression lines. Values are reported as the means of three biological replicates along with
standard deviation. The means with the same letters are not statistically significantly different. Statistical analysis of the results was conducted using
the one-way analysis of variance $(P<0.05)$

in the seed protein content was detected between these transgenic lines and the control (Additional file 1: Figure S2). These results indicate that expressing the KS domain can significantly increase both seed oil and seed mass in Arabidopsis.

\section{Effect of the KS domain expression on early seedling growth in Arabidopsis}

To examine the growth effects of the KS domain, the growth phenotype of the transgenic Arabidopsis plants was carefully observed during the life cycle. No obvious phenotypical difference could be detected between transgenic and untransformed wild-type control except for seed germination and early seedling growth. The seed germination rates of four transgenic lines were considerably higher $(217 \%, 205 \%, 211 \%$ and $203 \%)$ than that of the control (Fig. 7a). The lengths of 4-day seedlings of the transgenic lines were all longer compared to the wild type (Fig. 7b). This result indicated the expression of the KS domain from the PUFA synthase 

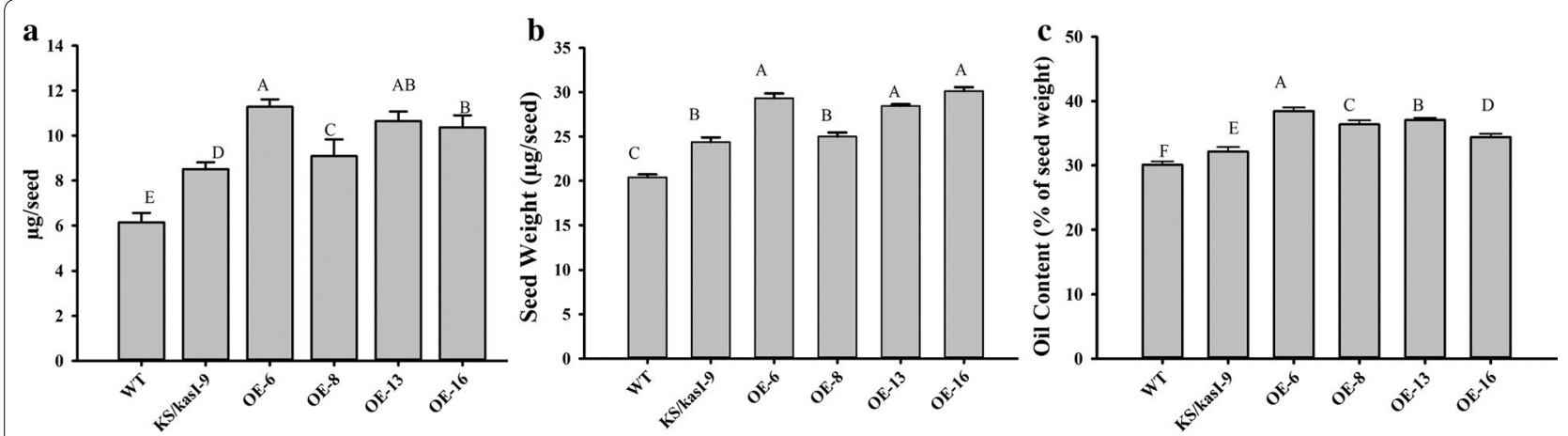

Fig. 6 The amount of oil, seed weight and oil content in transgenic seeds overexpressing the KS domain. a The amount of oil per seed in the T2 seeds of overexpression lines; $\mathbf{b}$ the seed weight of the transgenic lines; c seed oil content of the transgenic lines. WT wild type, KS/kas/ kas/ mutant complementation line, OE overexpression lines. Values are reported as the means of 10 biological replicates along with standard deviation. The means with the same letters are not statistically significantly different. Statistical analysis of the results was conducted using the one-way analysis of variance $(P<0.05)$

Table 1 Fatty acid composition (mol\%) in transgenic seeds overexpressing the KS domain

\begin{tabular}{lllllll}
\hline FA species & WT & KS/kasl-9 & OE-6 & OE-8 & OE-13 & OE-16 \\
\hline $16: 0$ & $12.05 \pm 0.83^{*}$ & $11.19 \pm 0.59^{*}$ & $10.07 \pm 0.20$ & $11.72 \pm 0.26^{*}$ & $10.27 \pm 0.39$ & $10.13 \pm 0.23$ \\
$16: 1-9$ & $1.66 \pm 0.20$ & $1.37 \pm 0.17$ & $0.74 \pm 0.03$ & $0.80 \pm 0.06$ & $0.66 \pm 0.05$ & $2.11 \pm 0.18^{*}$ \\
$18: 0$ & $10.73 \pm 1.32^{*}$ & $8.84 \pm 0.67$ & $7.29 \pm 0.38$ & $9.68 \pm 0.37^{*}$ & $8.38 \pm 0.33$ & $7.71 \pm 0.52$ \\
$18: 1-9$ & $11.19 \pm 0.82$ & $13.77 \pm 0.86$ & $17.38 \pm 0.70^{*}$ & $11.98 \pm 0.77$ & $17.93 \pm 0.61^{*}$ & $12.89 \pm 1.78$ \\
$18: 1-11$ & $2.26 \pm 0.23$ & $1.15 \pm 0.13$ & $1.90 \pm 0.12$ & $2.03 \pm 0.16$ & $2.81 \pm 0.23$ & $4.57 \pm 1.07^{*}$ \\
$18: 2$ & $20.81 \pm 0.74$ & $20.68 \pm 0.71$ & $20.49 \pm 0.56$ & $19.94 \pm 0.77$ & $20.50 \pm 0.41$ & $21.10 \pm 0.81$ \\
$18: 3$ & $14.19 \pm 1.23$ & $14.98 \pm 1.05$ & $14.35 \pm 0.53$ & $15.59 \pm 0.80$ & $12.30 \pm 0.26$ & $14.31 \pm 1.47$ \\
$20: 1-11$ & $14.20 \pm 0.54$ & $18.88 \pm 0.57^{* *}$ & $18.23 \pm 0.27^{* *}$ & $16.85 \pm 0.43^{*}$ & $16.20 \pm 0.38^{*}$ & $18.64 \pm 0.35^{* *}$ \\
Others & $13.91 \pm 1.12^{* *}$ & $9.14 \pm 0.77$ & $9.55 \pm 0.43$ & $11.38 \pm 0.73^{*}$ & $11.04 \pm 0.58^{*}$ & $8.51 \pm 1.21$ \\
C16-18 & $72.89 \pm 1.72$ & $71.98 \pm 1.17$ & $72.23 \pm 1.52$ & $71.74 \pm 3.19$ & $72.85 \pm 1.29$ & $72.81 \pm 1.77$ \\
SFA & $32.06 \pm 1.21^{* *}$ & $26.95 \pm 1.02$ & $23.03 \pm 0.73$ & $29.06 \pm 0.73^{*}$ & $24.72 \pm 1.11$ & $22.17 \pm 0.89$ \\
UFA & $67.94 \pm 1.73$ & $73.05 \pm 1.58^{*}$ & $76.97 \pm 0.82^{* *}$ & $70.94 \pm 1.13^{*}$ & $75.28 \pm 0.71^{* *}$ & $77.83 \pm 0.56^{* *}$ \\
UFA/SFA & 2.12 & 2.71 & 3.34 & 2.44 & 3.05 & 3.51 \\
\hline
\end{tabular}

The values represent means and standard deviation of four independent samples

FA fatty acid, WT wild type, KS/kasl kas/ mutant complementation line, OE overexpression lines, UFA unsaturated fatty acid, SFA saturated fatty acid, US/S refers to the ratio of unsaturated/saturated $F A$

The asterisk indicates significant difference in fatty acids composition between transgenic plants and WT $\left({ }^{*} P<0.05,{ }^{* *} P<0.01\right)$

in Arabidopsis could promote seed germination and seedling growth.

\section{Discussion}

In Thraustochytrium, the biosynthesis of VLCPUFAs is catalyzed by a PUFA synthase, a polyketide synthaselike mega-enzyme comprising three subunits each with multiple catalytic domains predicted on the characteristic active site residues [13]. However, the exact functions of these domains in the biosynthesis of these fatty acids are not well characterized. Our previous study indicated that a dissected KS domain from Subunit-B of the PUFA synthase from Thraustochytrium could complement the temperature-sensitive phenotype of $E$. coli $\beta$-ketoacylACP synthase I ( $F a b B)$ mutant and enhance the total fatty acid production in a wild-type E. coli strain [17]. In the present study, this domain was further functionally analyzed in A. thaliana. As the kasI mutant of Arabidopsis was defective in growth and development, and not amenable for re-transformation to express the KS domain for the complementation test, we resorted expressing the KS domain in Arabidopsis first and disrupting the endogenous KASI genes subsequently using a CRISPR/Cas9 system. Two homozygous insertion mutations of the target gene on the KS-expressed background were identified. Both resulted in the open reading frame shifting, 

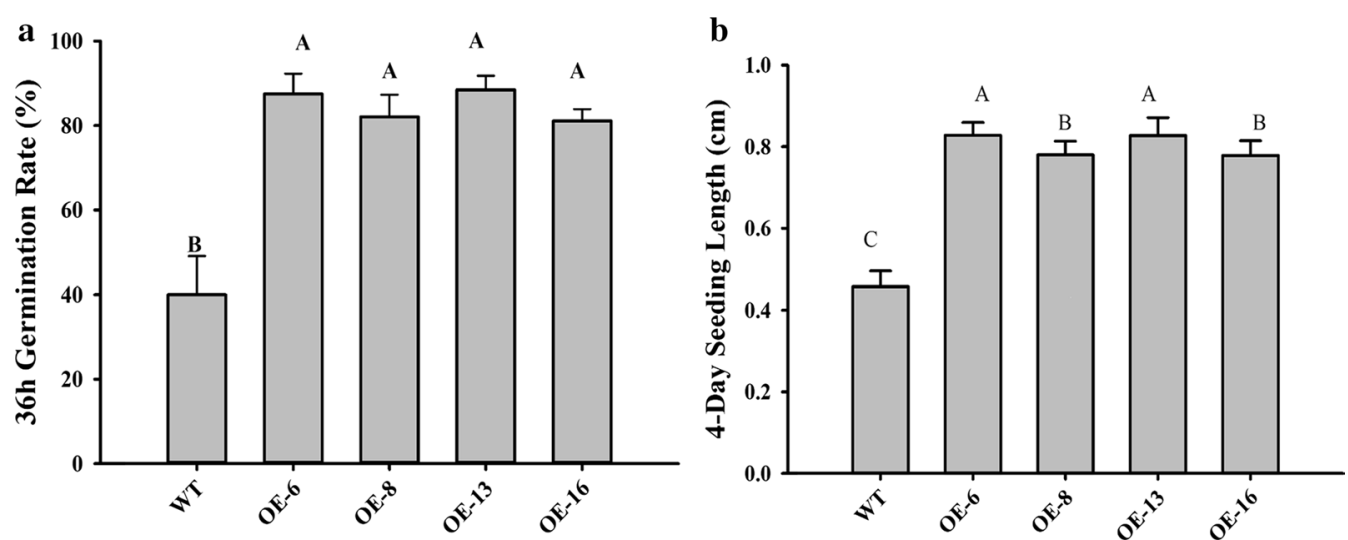

Fig. 7 Effect of KS domain expression on seed germination and early seedling growth in Arabidopsis. a Germination rates of wild type and transgenic lines at $36 \mathrm{~h}$ of imbibition. b The 4-day seedling length of WT and OE lines. WT wild type, OE overexpression lines. Values are reported as the means of 10 biological replicates along with standard deviation. The means with the same letters are not statistically significantly different. Statistical analysis of the results was conducted using the one-way analysis of variance $(P<0.05)$

which led to the inactivation of the KASI gene [25]. In the two complementation lines, the defective phenotypes of kasI knockout mutants such as small seedlings and dwarf plants were not observed anymore (Fig. 3). The RT-PCR analysis showed that transcription of the KS domain was detected in both leaves and developing seeds under the napin promoter, although the transcription level in developing siliques was 10 times higher than that in leaves. The similar expression pattern of the napin promoter has been seen in transgenic Brassica napus and tobacco [26, 27]. Nevertheless, the level of the KS domain expression under the napin promoter was enough for the functional complementation in Arabidopsis.

After the success of functional complementation, we then overexpressed the KS domain in wild-type Arabidopsis under the seed-specific promoter. The result showed that seed weight was increased by $20-40 \%$, and seed oil content was increased by $14 \%$ to $28 \%$ in four transgenic lines. The degree of the increase was coincided with the expression level of the KS domain in the siliques of these transgenic lines (Fig. 6). In addition, expression of the KS domain also had positive effects in seed germination and early seedling growth. This result suggests that the expression of the KS domain from the PUFA synthase could boost the de novo biosynthesis of fatty acids, thereby increasing the oil content in transgenic seeds.

In plant, the biosynthesis of long-chain fatty acids occurs in plastids by a type II fatty acid synthase complex comprising multiple discrete enzymes [10]. Among these enzymes, three $\beta$-ketoacyl-ACP synthases (KASI, KASII and KASIII) are key ones responsible for the condensing reaction, the first of four chemical reactions in a repetitive cycle of fatty acid synthesis by the addition of a two-carbon unit a time donated by malonyl-ACP. KASIII catalyzes the first condensation of fatty acid synthesis between acetyl-CoA/ACP and malonyl-ACP, giving a $\mathrm{C} 4$ acyl chain. KASI ( $\beta$-ketoacyl-ACP synthase I) catalyzes the subsequent condensation reactions from $\mathrm{C} 4$ to $\mathrm{C} 16$ acyl chains. KASII catalyzes the final condensation of fatty acid synthesis producing a $\mathrm{C} 18$ acyl chain. After synthesis, long-chain fatty acids are modified in cytosol and assembled into storage glycerolipid triacylglycerols [28]. Previous studies showed that out of three KAS enzymes, KASI might be the most important in regulating fatty acid biosynthesis in plant. Knocking out/down of KASI gene could significantly reduce fatty acid production, while overexpression of it could increase seed weight and total fatty acids in seeds $[29,30]$. Here, we showed that overexpression of a KS domain from the PUFA synthase of protist Thraustochytrium could increase seed weight by $20-40 \%$ and total fatty acids by $48-80 \%$ per seed relative to the wild-type control in Arabidopsis, which are much higher than those in the overexpression of plant endogenous KASI [30]. The reason for the difference in the two expressions remains to be determined. It is possible that the KS domain protein from the PUFA synthase might simply possess higher condensation activity. In addition, expression of a KS domain of PUFA synthases of microbial origin in plant might own an advantage in minimizing the possible feedback inhibition over the expression of an endogenous KASI [31].

In the past few years, several molecular strategies have been attempted to increase oil production in oilseed crops. One strategy is to increase fatty acid assembly into triacylglycerols by overexpressing acyl-CoA acyltransferase in the Kennedy pathway. For instance, overexpression of lysophosphatidic acid acyltransferase (LPAAT) or DGAT1 significantly increases oil contents in seeds. Fatty 
acid profile in the oil is determined by substrate specificity of acyl-CoA acyltransferases used for fatty acid acylations [6,32]. Another strategy is to increase the synthetic efficiency of fatty acids prior to the assembly by overexpression of enzymes directly involved in the biosynthetic process. For instance, overexpression of ACCase resulted in an increase in oil content [7,33-35]. In this strategy, fatty acid profile of the oil would be similar to that of wild type. In the present study, expression of the KS domain from the PUFA synthase significantly enhanced oil content in transgenic lines and at the same time the fatty acid profile of seed oil was also altered to the higher proportion of unsaturated fatty acids. This result indicates the condensation process, particularly the condensation steps from $\mathrm{C} 4$ to $\mathrm{C} 16$, during the fatty acid biosynthesis might be the limiting step, and overexpression of the KS domain of the Thraustochytrium PUFA synthase, although it only shares about $20 \%$ amino acid identity to Arabidopsis KASI, can increase seed oil and seed mass simultaneously in Arabidopsis. The reason why the unsaturated level of fatty acids was also increased in the transgenics remains to be determined. It is probably due to the unique activity of the KS domain of microbial origin. Overexpression of the domain may not only enhance the main condensation reactions in fatty acid synthesis, but also stimulate the activity of downstream fatty acidmodifying enzymes such as acyl-ACP and phospholipid desaturases and FAE-type elongases. Collectively, our research in the enhanced condensation activity by overexpression of the KS domain from protist PUFA synthase resulting in significantly increased seed weight and seed oil offers a new approach to increase oil production in oilseed plants.

\section{Conclusions}

Seed oil is an important renewable bioresource for food and bioproducts. This research provides a new strategy to enhance oil and unsaturated fatty acid production by expressing KS domains from polyketide synthase-like PUFA synthases of microbial origin in oilseed crops for nutritional and industrial uses.

\section{Methods}

\section{Materials}

Agrobacterium and E. coli media were purchased from Bio Basic Inc. (York, Ontario, Canada). Fatty acids and their standards were obtained from Nu-Chek-Prep, Inc. (Elysian, MN, USA). Q5 DNA polymerase, restriction enzymes, T7 Endonuclease and deoxynucleotide triphosphate (dNTP) were purchased from New England Biolabs (Ipswich, MA, USA). HP Taq DNA polymerase was obtained from Bio Basic Inc. (York, Ontario, Canada). Primers were synthesized from Sigma-Aldrich (St.
Louis, MO, USA). GC grade solvents were from Fisher Scientific (Ottawa, ON, Canada). All other chemicals were purchased from Sigma-Aldrich Ltd (Oakville, ON, Canada). The intermediate vector and expression vector for CRISPR/Cas9 system were purchased from Addgene (Watertown, MA, USA).

\section{Plant materials and growth conditions}

Wild-type (Col-0) Arabidopsis (A. thaliana) seeds were surface-sterilized and remained in darkness for 3 days at $4{ }^{\circ} \mathrm{C}$. Seeds were germinated on a halfstrength MS medium [36], and the plates were placed in a growth chamber set to $22{ }^{\circ} \mathrm{C}$ under a 16-h-light $\left(120 \mu \mathrm{Em}^{-2} \mathrm{~s}^{-1}\right) / 8$-h-dark photoperiod. After 12 days, each seedling was transplanted to pots. Individual plants in each pot were arranged randomly in a tray. When plants began to flower, a 60-cm stick was inserted in each pot to tie the stems, and at maturity, the pot was placed inside an upright rectangular transparent, perforated plastic bag $(60 \times 6 \mathrm{~cm})$.

\section{Construction of plant expression vectors}

To express the KS domain in A. thaliana, two different constructs with different selection and screening marker genes were built. The plastidial overexpression construct contains a KS domain from PUFA synthase (accession no. PRJNA368972) fused with a functional chloroplasts transit peptide (CTP) at the N-terminus [37] and a DsRed2 from Discosoma $s p$. encoding a red fluorescent protein, each under the control of a seed-specific napin promoter [38], as well as an antibiotic kanamycin-resistance gene under the control of a constitutive nos promoter.

For disrupting Arabidopsis KASI (AtKASI), a CRISPR/ Cas9 construct targeting AtKASI was built. Two single guide RNAs (sgRNA) targeting KASI gene (AT5G46290) were designed using the previous protocol [website: (http://skl.scau.edu.cn)] [39]. The sgRNA sequences were then inserted into an intermediate plasmid behind an AtU3d promoter. The recombinant AtU3d promoterdriving sgRNA cassettes were then inserted into pYLCRISPR/Cas9P ${ }_{\text {ubi }}-\mathrm{B}$ vector [21]. For transgenic seed screening, a napin promoter was used to guide a modified green fluorescent protein (eGFP) gene at the SpeI site of pYLCRISPR/Cas9P ${ }_{\text {ubi }}{ }^{-B}$.

Transformation of Agrobacterium with recombinant vector Electrocompetent cells of Agrobacterium tumefaciens GV3101 were prepared as follows. Agrobacterium cells were grown for $24 \mathrm{~h}$ in $\mathrm{LB}$ medium with $50 \mu \mathrm{g} / \mathrm{mL}$ gentamicin. When the $\mathrm{A}_{600}$ reached 0.7, the cells were chilled on ice and pelleted by centrifugation ( $5000 \mathrm{rpm}$ for $10 \mathrm{~min}$ at $4{ }^{\circ} \mathrm{C}$ ). The pellet was washed in $1,0.5$ and 0.02 volumes of cold $10 \%(\mathrm{v} / \mathrm{v})$ sterile glycerol and 
resuspended in 0.01 volume of cold $10 \%(\mathrm{v} / \mathrm{v})$ glycerol. The electrocompetent cells were then frozen in liquid $\mathrm{N}_{2}$ and stored at $\leq-70{ }^{\circ} \mathrm{C}$. The Agrobacterium cells were transformed by electroporation with 20 to $50 \mathrm{ng}$ of DNA according to the manufacturer's instructions. Transformed cells were plated on a selective medium (LB agar with corresponding antibiotic), and incubated for $48 \mathrm{~h}$ at $28^{\circ} \mathrm{C}$. Transformed cells were grown for $16 \mathrm{~h}$ $\left(28{ }^{\circ} \mathrm{C}, 225 \mathrm{rpm}\right)$ in $5 \mathrm{~mL} \mathrm{LB}$ broth with corresponding antibiotic and $50 \mu \mathrm{g} / \mathrm{mL}$ gentamicin. The fidelity of the construct was rechecked by PCR before floral dipping transformation.

\section{Transformation of Arabidopsis and genotyping of transgenics}

Arabidopsis at the flowering stage grown in the growth chamber was used for transformation using the floral dip method [40]. The transgenic seeds were selected by fluorescence and grown on soil for the next generation. The transgenic plants were genotyped every generation, and the seeds from positive lines were harvested and planted for the next generation. Genomic DNA of wild-type and transgenic plants was extracted with Edward method [41]. The PCR condition was 30 cycles of denaturation at $95{ }^{\circ} \mathrm{C}$ for $30 \mathrm{~s}$, annealing at $60{ }^{\circ} \mathrm{C}$ for $30 \mathrm{~s}$, and elongation at $72{ }^{\circ} \mathrm{C}$ for $30 \mathrm{~s}$ with primers F-KASI-1-seq and R-KASIseq (Additional file 1: Table S1).

\section{Transcriptional expression analysis}

The samples of 7 th-8th rosette leaf and developing siliques at 12 to 14 days after flowering (DAF) from T3 overexpressing lines and wild type were collected and frozen in liquid $\mathrm{N}_{2}$. RNA was extracted by Qiagen RNeasy Mini Kit (Germantown, MD, USA) and treated with DNase I for $30 \mathrm{~min}$ to digest contaminating DNA in samples. The biosynthesis of complementary DNA was carried out using SuperScript ${ }^{\text {TM }}$ III Reverse Transcriptase from Invitrogen (Carlsbad, CA, USA). Quantitative RTPCR was performed with PowerUp ${ }^{\mathrm{TM}} \mathrm{SYBR}^{\mathrm{TM}}$ Green Master Mix from Fisher Scientific (Carlsbad, CA, USA). The PCR conditions were as follows: $50{ }^{\circ} \mathrm{C} 2 \mathrm{~min}, 95{ }^{\circ} \mathrm{C}$ for $2 \mathrm{~min}, 40$ cycles of $95^{\circ} \mathrm{C}$ for $5 \mathrm{~s}, 60{ }^{\circ} \mathrm{C}$ for $1 \mathrm{~min}$, and one cycle of $95^{\circ} \mathrm{C}$ for $15 \mathrm{~s}, 60{ }^{\circ} \mathrm{C}$ for $1 \mathrm{~min}$, and $95^{\circ} \mathrm{C}$ for $15 \mathrm{~s}$ for melt curve stage. The expression of the housekeeping gene AtActin-2 (AT3G18780) was used as references [42]. The expression level was normalized by that of Actin-2, and the primer pairs used for the PCR are listed in Additional file 1: Table S1.

\section{Fatty acid composition and oil content analysis}

For fatty acid analysis, single seed was placed in a glass tube with a screw cap and $5 \mu \mathrm{L}$ of $10 \mu \mathrm{g} / \mathrm{mL}$ heptanoic acid (C17:0) was added as an internal standard for quantification. Two milliliters of $1 \%(\mathrm{v} / \mathrm{v}) \mathrm{H}_{2} \mathrm{SO}_{4}$ in methanol was added to the glass tube containing seed and internal standard. Sample was heated for $10 \mathrm{~min}$ at $80^{\circ} \mathrm{C}$, and seed was crushed by glass rod and incubated at $80^{\circ} \mathrm{C}$ for $1 \mathrm{~h}$ to transmethylate fatty acids into their FAMEs. FAMEs were extracted with $2 \mathrm{~mL}$ of hexane and $1 \mathrm{~mL}$ of $0.9 \% \mathrm{NaCl}$. The total fatty acid profiles and quantity were analyzed on an Agilent 7890A system installed with a DB-23 column (30 m, $0.25 \mathrm{~mm}, 0.25 \mu \mathrm{m})[13,17]$.

\section{Seed germination and seedling growth}

The surface-sterilized seeds were plated on agar plates of MS medium containing 1\% sucrose and incubated in darkness for 3 days at $4{ }^{\circ} \mathrm{C}$. The plates were then placed vertically in a growth chamber set to $22{ }^{\circ} \mathrm{C}$ under a 16-h-light $\left(120 \mu \mathrm{Em}^{-2} \mathrm{~s}^{-1}\right) / 8$-h-dark photoperiod for 4 days. Then, the germination rate and seedling length were measured.

\section{CRISPR/Cas9 knockout mutant screening and complementation test}

The knockout mutant generated by CRISPR/Cas9 was genotyped in T2 plants by sequencing of target genes. The authentic mutant lines were used for the complementation test. The phenotype of the mutant and mutant complemented lines were observed and recorded every week.

\section{Additional file}

Additional file 1: Table S1. Primers used for this study. Figure S1. Comparison of sequences surrounding the sgRNA sequences in the KASI genes. KASI, original KASI; kasl, kasl knockout mutant; KS/kasl, kas/ knockout mutant with KS domain; Blue boxes represent PAM sites, and Green boxes represent the target sites. Figure $\mathbf{S 2}$. Protein content in transgenic seeds overexpressing the KS domain. WT, wild type; $\mathrm{OE}$, overexpression lines. Values are reported as the means of 4 biological replicates along with standard deviation. The means with the same letters are not statistically significantly different. Statistical analysis of the results was conducted using the one-way analysis of variance $(P<0.05)$.

\section{Acknowledgements}

We thank Addgene (Addgene Headquarters, Watertown, MA, USA) for providing intermediate vector and expression vector for CRISPR/Cas9 knockout system.

\section{Authors' contributions}

$X X, D M$ and $X Q$ conceived and designed the experiments; XX performed most of the experiments; DM provided technical assistance to $X X ; X X$ analyzed the data; XX, DM and XQ wrote the article. All authors read and approved the final manuscript.

\section{Funding}

This research was supported by Natural Sciences and Engineering Research Council of Canada. 


\section{Availability of data and materials}

All data generated or analyzed during this study are included in this article and its Additional file.

\section{Ethics approval and consent to participate}

Not applicable.

\section{Consent for publication}

Not applicable.

\section{Competing interests}

The authors declare that they have no competing interests.

Received: 13 March 2019 Accepted: 21 June 2019

Published online: 29 June 2019

\section{References}

1. Robinson LE, Mazurak VC. $n-3$ polyunsaturated fatty acids: relationship to inflammation in healthy adults and adults exhibiting features of metabolic syndrome. Lipids. 2013;48:319-32.

2. Wang W, Zhu J, Lyu F, Panigrahy D, Ferrara KW, Hammock B, et al. Omega-3 polyunsaturated fatty acids-derived lipid metabolites on angiogenesis, inflammation and cancer. Prostaglandins Other Lipid Mediat. 2014;113-115:13-20.

3. Kumar A, Sharma A, Upadhyaya K. Vegetable oil: nutritional and industrial perspective. Curr Genom. 2016;17:230-40.

4. Liu J, Hua W, Zhan G, Wei F, Wang X, Liu G. Increasing seed mass and oil content in transgenic Arabidopsis by the overexpression of wri1-like gene from Brassica napus. Plant Physiol Biochem. 2010:48:9-15.

5. Vigeolas H, Waldeck P, Zank T, Geigenberger P. Increasing seed oil content in oil-seed rape (Brassica napus L.) by over-expression of a yeast glycerol-3-phosphate dehydrogenase under the control of a seed-specific promoter. Plant Biotechnol J. 2007:5:431-41.

6. Jako C. Seed-specific over-expression of an Arabidopsis cDNA encoding a diacylglycerol acyltransferase enhances seed oil content and seed weight. Plant Physiol. 2001;126:861-74

7. GuY, He L, Zhao C, Wang F, Yan B, Gao Y, et al. Biochemical and transcriptional regulation of membrane lipid metabolism in maize leaves under low temperature. Front Plant Sci. 2017;8:1-13.

8. Zhang Y, Yu L, Yung KF, Leung DY, Sun F, Lim BL. Over-expression of AtPAP2 in Camelina sativa leads to faster plant growth and higher seed yield. Biotechnol Biofuels. 2012;5:1-10.

9. Roesler K, Shintani D, Savage L, Boddupalli S, Ohlrogge J. Targeting of the Arabidopsis homomeric acetyl-coenzyme A carboxylase to plastids of rapeseeds. Plant Physiol. 1997;113:75-81.

10. Magnuson K, Jackowski S, Rock CO, Cronan JE. Regulation of fatty acid synthesis. Biochem Soc Trans. 1993;57:522.

11. Meesapyodsuk D, Ye S, Chen Y, Chen Y, Chapman RG, Qiu X. An engineered oilseed crop produces oil enriched in two very long chain polyunsaturated fatty acids with potential health-promoting properties. Metab Eng. 2018:49:192-200.

12. Zhao XM, Meesapyodsuk D, Qu CM, Qiu X. Genomic analysis of genes involved in the biosynthesis of very long chain polyunsaturated fatty acids in Thraustochytrium sp. 26185. Lipids. 2016;51(9):1065-75.

13. Meesapyodsuk D, Qiu X. Biosynthetic mechanism of very long chain polyunsaturated fatty acids in Thraustochytrium sp. 26185. J Lipid Res. 2016:57(10):1854-64.

14. Schweizer E, Hofmann J. Microbial type I fatty acid synthases (FAS): major players in a network of cellular FAS systems. Microbiol Mol Biol Rev. 2004;68:501-17.

15. Yoshida K, Hashimoto M, Hori R, Adachi T, Okuyama H, Orikasa Y, et al. Bacterial long-chain polyunsaturated fatty acids: their biosynthetic genes, functions, and practical use. Mar Drugs. 2016;14:94.

16. Janßen HJ, Steinbüchel A. Fatty acid synthesis in Escherichia coli and its applications towards the production of fatty acid based biofuels. Biotechnol Biofuels. 2014;7:1-26.

17. Xie X, Meesapyodsuk D, Qiu X. Ketoacylsynthase domains of a PUFA synthase in Thraustochytrium can effectively function as stand-alone enzymes in Escherichia coli. Appl Environ Microbiol. 2017:83(9):e03133-16.

18. Zhang L, Joshi AK, Hofmann J, Schweizer E, Smith S. Cloning, expression, and characterization of the human mitochondrial $\beta$-ketoacyl synthase: complementation of the yeast cem1 knock-out strain. J Biol Chem. 2005;280(13):12422-9.

19. White SW, Zheng J, Zhang Y-M, Rock CO. The structural biology of type II fatty acid biosynthesis. Annu Rev Biochem. 2005;74:791-831.

20. Davies C, Heath RJ, White SW, Rock CO. The $1.8 \AA$ crystal structure and active-site architecture of $\beta$-ketoacyl-acyl carrier protein synthase III (FabH) from Escherichia coli. Structure. 2000;8(2):185-95.

21. Ma XL, Liu YG. CRISPR/Cas9-based multiplex genome editing in monocot and dicot plants. Curr Protoc Mol Biol. 2016;115:1-21.

22. Wu GZ, Xue HW. Arabidopsis $\beta$-ketoacyl-[acyl carrier protein] synthase I is crucial for fatty acid synthesis and plays a role in chloroplast division and embryo development. Plant Cell. 2010;22:3726-44.

23. Baud S, Dubreucq B, Miquel M, Rochat C, Lepiniec L. Storage reserve accumulation in Arabidopsis: metabolic and developmental control of seed filling. Arabidopsis Book. 2008;6:e0113.

24. Lemieux B, Miquel M, Somerville C, Browse J. Mutants of Arabidopsis with alterations in seed lipid fatty acid composition. Theor Appl Genet. 1990;80(2):234-40.

25. Schiml S, Puchta H. Revolutionizing plant biology: multiple ways of genome engineering by CRISPR/Cas9. Plant Methods. 2016:12:1-9.

26. Ellerström M, Stålberg K, Ezcurra I, Rask L. Functional dissection of a napin gene promoter: identification of promoter elements required for embryo and endosperm-specific transcription. Plant Mol Biol. 1996;32:1019-27

27. Kjell S, Mats E, Staffan S, Ines E, Paul W, Lars R. Heterologous and homologous transgenic expression directed by a $2 S$ seed storage promoter of Brassica napus. CEUR Workshop Proc. 2016;1621:36-43.

28. Baud S, Lepiniec L. Physiological and developmental regulation of seed oil production. Prog Lipid Res. 2010;49:235-49.

29. Yang T, Xu R, Chen J, Liu A. $\beta$-ketoacyl-acyl carrier protein synthase (KASI) plays crucial roles in the plant growth and fatty acids synthesis in tobacco. Int J Mol Sci. 2016;17(8):E1287.

30. Ding W, Lin L, Zhang B, Xiang X, Wu J, Pan Z, et al. OsKASI, a $\beta$-ketoacyl[acyl carrier protein] synthase I, is involved in root development in rice (Oryza sativa L.). Planta. 2015;242:203-13.

31. Ke X, Zou W, Ren Y, Wang Z, Li J, Wu X, et al. Functional divergence of chloroplast Cpn60a subunits during Arabidopsis embryo development. PLoS Genet. 2017:13:1-27.

32. Savadi S, Naresh V, Kumar V, Bhat SR. Seed specific overexpression of Arabidopsis DGAT1 in india mustard (Brassica juncea) increases seed oil and seed weight. Botany. 2015;184:1-27.

33. Cui Y, Liu Z, Zhao Y, Wang Y, Huang Y, Li L, et al. Overexpression of heteromeric GhACCase subunits enhanced oil accumulation in upland cotton. Plant Mol Biol Rep. 2017;35:287-97

34. Shintani D, Roesler K, Shorrosh B, Savage L, Ohlrogge J. Antisense expression and overexpression of biotin carboxylase in tobacco leaves. Plant Physiol. 1997;114:881-6.

35. Thelen JJ, Ohlrogge JB. Both antisense and sense expression of biotin carboxyl carrier protein isoform 2 inactivates the plastid acetyl-coenzyme A carboxylase in Arabidopsis thaliana. Plant J. 2002;32:419-31.

36. Murashige T, Skoog F. A revised medium for rapid growth and bio assays with tobacco tissue cultures. Physiol Plantarum. 1962;15:473-97.

37. Lee DW. Functional characterization of sequence motifs in the transit peptide of Arabidopsis small subunit of Rubisco. Plant Physiol. 2006;140:466-83.

38. Josefsson LG, Lenman M, Ericson ML, Rask L. Structure of a gene encoding the $1.7 \mathrm{~S}$ storage protein, napin, from Brassica napus. J Biol Chem. 1987:262(25):12196-201.

39. Xie X, Ma X, Zhu Q, Zeng D, Li G, Liu YG. CRISPR-GE: a convenient software toolkit for CRISPR-based genome editing. Mol Plant. 2017;10:1246-9.

40. Clough SJ, Bent AF. Floral dip: a simplified method for Agrobacterium-mediated transformation of Arabidopsis thaliana. Plant J. 1998;16(6):735-43.

41. Edwards K, Johnstone C, Thompson C. A simple and rapid method for the preparation of plant genomic DNA for PCR analysis. Nucleic Acids Res. 1991:19(6):1349. 
42. Wang H, Wang J, Jiang J, Chen S, Guan Z, Liao Y, et al. Reference genes for normalizing transcription in diploid and tetraploid Arabidopsis. Sci Rep. 2014;4:1-8

\section{Publisher's Note}

Springer Nature remains neutral with regard to jurisdictional claims in published maps and institutional affiliations.
Ready to submit your research? Choose BMC and benefit from:

- fast, convenient online submission

- thorough peer review by experienced researchers in your field

- rapid publication on acceptance

- support for research data, including large and complex data types

- gold Open Access which fosters wider collaboration and increased citations

- maximum visibility for your research: over $100 \mathrm{M}$ website views per year

At BMC, research is always in progress.

Learn more biomedcentral.com/submissions 